\title{
BOUNDARY SETTING IN SIMULATING GREENHOUSE VENTILATION BY FLUENT SOFTWARE
}

\author{
Cuiping Hou ${ }^{1,2}$, Chengwei Ma, ${ }^{1,2 *}$ \\ ${ }^{I}$ College of Water Conservancy and Civil Engineering, China Agricultural University, Beijing, \\ Beijing, China, 100083 \\ ${ }^{2}$ Bia-Environmental Engineering Lab. Key Lab of Mart., 100083, China \\ * Corresponding author, Address: P.O. Box 195, China Agricultural University, 17 Tsinghua \\ East Road, Beijing, 100083, P. R. China, Tel: +86-10-62736413, Fax: +86-10-62736413, \\ Email:macwbs@cau.edu.cn
}

\begin{abstract}
Fluent, as one of the commercial CFD packages, the author discussed its application in greenhouse ventilation research. In the paper, the under problems would be expounded: in fluent what models will be used in greenhouse studying, and the details during in these models used, and the comparing of the same style models. The steps to solve the greenhouse problem also was expatiate in this paper, mainly including: how to abstract its physical models; how to define its compute domains; the factor should be considered in creating and meshing the geometry; the types of boundary condition, and how to give the boundary conditions, in this section, the author will take some instances to emphasize discuss; the selection of parameter in simulation, and a few of methods of post processing. At the last, the author introduced some even existing problem in simulating.
\end{abstract}

Keywords: fluent, simulation, boundary condition

Hou, C. and Ma, C., 2008, in IFIP International Federation for Information Processing, Volume 259; Computer and Computing Technologies in Agriculture, Vol. 2; Daoliang Li; (Boston: Springer), pp. 1391-1395. 


\section{THE PROBLEM OF GREENHOUSE VENTILATION CAN BE SOLVED BY FLUENT}

\subsection{Abstract of the Physical Models}

To a problem of ventilation in greenhouse, at first we should make some assumption to determine a physical model. Subsequent factors should be think about in this process: two-dimensional or three-dimensional; steadystate or unsteady-state; laminar flow or turbulence flow; physical parameters, change or unchanged, compressible or uncompressible, uniform or not uniform in speed; the style of the boundary condition. All computations were performed assuming conditions.

\subsection{Computational Domain of the Simulation}

The size of the greenhouse model is significantly limited by the available computer memory and processor speed. The solution domain is subdivided into a finite number of contiguous control volumes. Usually we selected a large domain including the greenhouse, and the determine of the size of the computational domain need our try, the result of the simulation should be no more than significant improvement (Kacira et al., 1998; Mistriotis et al., 1997; Short, 1996).

\subsection{The Grid}

The CFD model calculating time was strongly dependent on the size of the grid domain and a node adjustment factor was used to minimize grid numbers. The grid size should be dense enough to describe accurate flows around complex shapes and sparse enough to minimize calculations in uniform flow areas. The relatively small accuracy increase between the different grids indicates that the grid dependency on the solution has become minimal. It results from an empirical compromise between a dense grid, associated with a long computational time, and a less dense one, associated with a small deterioration of the simulated results. Maximum level allowed by the software; In the case of the greenhouse with crop the necessary grid refinement and the problems of convergence did not allow a reduction in the number of elements; In the case of the empty greenhouse a diminution in the number of elements did not cause a significant variation in the accuracy of 
simulations, neither it produce convergence problems. From the general grid specifications, a body-fitted coordinate (BFC) grid method was used with fluent to acquire the most accurate flow patterns and computations at the boundaries of the greenhouse. Smooth and uniform enough to guarantee the best results. The discretisation is performed with triangular and quadrilateral elements. The grid was an unstructured grid with higher density at the vent openings, where the flow was subject to strong gradients.

The scale of detail which should be applied must be investigated to achieve a good balance between increased complexity, demanding modeling skills and computational effort, and the required accuracy of the model output.

\section{BOUNDARY CONDITIONS}

The choice of suitable numerical parameters and sufficiently accurate boundary conditions required for accurate results. The velocities are obtained from the conservation principle, the pressure is obtained from the conservation of mass principle and the temperature is obtained from the law of conservation of energy. Crop canopy, the flow would take into account the resistance presented by the canopy, the heat balance would include effects of transpiration, convection and radiation and mass balance would include the effect of photosynthesis on carbon dioxide levels, transpiration and convectional. Boundary conditions contain the effects of external factors on the flow and temperature.

FLUENT required input variables associated with the properties of the air and boundary conditions. The dynamic boundary conditions prescribed a null pressure gradient in the air, at the limits of the computational domain, and wall-type boundary conditions along the floor, the walls and the roof and vents surfaces of the scale greenhouse model. The thermal boundary conditions imposed fixed temperatures at the limits of the computational domain and at roof and floor levels, and adiabatic conditions along the side walls of the scale greenhouse model. The dynamic boundary conditions prescribed a null pressure gradient in the air, at the limits of the computational domain, and wall-type boundary conditions along the floor, the walls and the roof and vents surfaces of the scale greenhouse model. The thermal boundary conditions imposed fixed temperatures at the limits of the computational domain and at roof and floor levels, and adiabatic conditions along the side walls of the scale greenhouse model.

The values of those inputs were chosen from the fluent manual and from the experimental greenhouse. A list of the input values usually is: momentum factors, inlet velocity or inlet profile, model constants, inlet 
kinetic energy, outer boundary conditions (usually be setting as fixed pressure), if think about the plant, crop porosity should be taken account; energy factors, side wall temperature (usually be setting as adiabatic), cover/soil temperature, air temperature, heat flux from floor/roof, sky temperature, radioactive heat transfer; mass factors should setting air properties, include, specific heat, thermal conductivity, dynamic viscosity, atmospheric pressure, also include the aid density, which can be set as ideal gas, also can be set as boussinesq, in this case the density becomes constant in all equations except for the buoyancy term in the momentum equations. Fluid flow, heat and mass transfer processes, once all inputs were defined in fluent, a case file was generated. Fluent utilized the case file to solve conservation equations using the SIMPLE algorithm with an iterative lineby-line solver and a multi-grid acceleration. When the numerical solutions were converged, a numerical and graphical output of the results is obtained.

If only ventilation due to the wind is studied, the gravity is considered zero, the heat input for this situation can be set to any value not influencing the ventilation rate. If the buoyancy effect is included, the heat input was set to a value equal to the heat supplied by solar radiation and gravity is reset.

Body-fitted coordinates, which is a coordinate system with grid lines that coincide with the boundaries of the geometry. Body-fitted coordinates to acquire the most accurate flow patterns and computations at the boundaries (Fluent, 1993). Body-fitted coordinates were also applied to exactly conform the grid to the contours of the boundary conditions.

To limit the control volume of the large hall containing the reduced-scale greenhouse, pressure-type boundary conditions were selected for the ambient air. These boundary conditions prescribe fixed pressure and temperature conditions at the limits of the computational domain and the inlet and outlet air velocities are automatically computed to satisfy the continuity conditions.

\section{DISCUSSION}

Until now, because of the great variability of the climatic parameters involved nobody has found a model of greenhouse ventilation for general application (Bailey et al., 1999). CFD can be a useful tool in the study of in the internal greenhouse climate. With a CFD model a 'virtual reality' simulation may be created that is very versatile and relatively cheap. But simulation result is not so believable, in many times, its result even need validate, present methods include, sonic anemometers and smoke tracing techniques, which can measure and visualize the internal airflow, these and other tools like PIV, hot-wire anemometers, laser-Doppler anemometers, 
thermo-couples, carbon dioxide monitors and other specialized equipment. Future applications of fluent even lie in conducting parametric studies.

\section{REFERENCES}

Bailey, B.J. 1999. Constraints limitations and achievements in greenhouse natural ventilation. Acta Hortic, 534, 21-30.

Kacira, M., Short, T.H., Stowell, R.R. 1998. A CFD evaluation of naturally ventilated multispan, sawtooth greenhouses. Trans. ASAE, 41 (3), 833-836.

Mistriotis, A., De Jong I. 1997. Computational fluid dynamics study as a tool for the analysis of ventilation and indoor microclimate in agricultural building. Neth. J. Agric. Sci. 45, 81-96.

Short I.H. 1996. Dynamic model of naturally ventilated poly houses, Grower Talks Magazine, summer. 